\title{
Battery Health Estimation for loT Devices using V-Edge Dynamics
}

\author{
Kumar, Arjun
}

ACM

2020-03-04

Kumar , A , Hoque , M A , Nurmi , P , Pecht , M G , Tarkoma , S \& Song , J 2020 , Battery Health Estimation for loT Devices using V-Edge Dynamics . in Proceedings of the $21 \mathrm{st}$ International Workshop on Mobile Computing Systems and Applications : HotMobile'2020 . ACM , New York , pp. 56-61, HotMobile '20: The 21st International Workshop on Mobile Computing Systems and Applications , Austin , United States , 03/03/2020 . https://doi.org/10.1145/3376897.337785

http://hdl.handle.net/10138/321931

https://doi.org/10.1145/3376897.3377858

acceptedVersion

Downloaded from Helda, University of Helsinki institutional repository.

This is an electronic reprint of the original article.

This reprint may differ from the original in pagination and typographic detail.

Please cite the original version. 


\section{Battery Health Estimation for IoT Devices using V-edge Dynamics}

\author{
Arjun Kumar \\ KAIST, Republic of Korea \\ arjun@nclab.kaist.ac.kr \\ Michael G. Pecht \\ University of Maryland, USA \\ pecht@umd.edu
}

\author{
Mohammad A. Hoque \\ University of Helsinki, Finland \\ mohammad.a.hoque@helsinki.fi \\ Sasu Tarkoma \\ University of Helsinki, Finland \\ sasu.tarkoma@helsinki.fi
}

\author{
Petteri Nurmi \\ University of Helsinki, Finland \\ petteri.nurmi@helsinki.fi \\ Junehwa Song \\ KAIST, Republic of Korea \\ junesong@nclab.kaist.ac.kr
}

\begin{abstract}
Deployments of battery-powered IoT devices have become ubiquitous, monitoring everything from environmental conditions in smart cities to wildlife movements in remote areas. How to manage the life-cycle of sensors in such large-scale deployments is currently an open issue. Indeed, most deployments let sensors operate until they fail and fix or replace the sensors post-hoc. In this paper, we contribute by developing a new approach for facilitating the life-cycle management of large-scale sensor deployments through online estimation of battery health. Our approach relies on so-called $V$-edge dynamics which capture and characterize instantaneous voltage drops. Experiments carried out on a dataset of battery discharge measurements demonstrate that our approach is capable of estimating battery health with up to $80 \%$ accuracy, depending on the characteristics of the devices and the processing load they undergo. Our method is particularly well-suited for the sensor devices, operating dedicated tasks, that have constant discharge during their operation.
\end{abstract}

\section{CCS CONCEPTS}

- Computer systems organization $\rightarrow$ Embedded systems; • Hardware $\rightarrow$ Batteries; Sensor applications and deployments.

\section{KEYWORDS}

Lithium Battery, Power Models, Battery Health, Battery Capacity, Internet of Things

\section{ACM Reference Format:}

Arjun Kumar, Mohammad A. Hoque, Petteri Nurmi, Michael G. Pecht, Sasu Tarkoma, and Junehwa Song. 2020. Battery Health Estimation for IoT Devices using V-edge Dynamics. In Proceedings of ACM Conference (Conference'17). ACM, New York, NY, USA, 6 pages. https://doi.org/10.1145/ nnnnnnn.nnnnnnn

\section{INTRODUCTION}

Every year millions of battery-powered IoT devices are being deployed, monitoring everything from environmental conditions [12] to occupant health conditions in smart homes [5], animal behavior in the wild [2], and beyond. Battery life-cycle management is critical for long-term operation of these deployments as many of the devices are rarely - or never charged [5] - or rely on intermittent charging, e.g., through energy harvesting [11]. At the same time, they operate in highly dynamic environments, ranging from stable room temperature to extreme weather conditions in the wild.

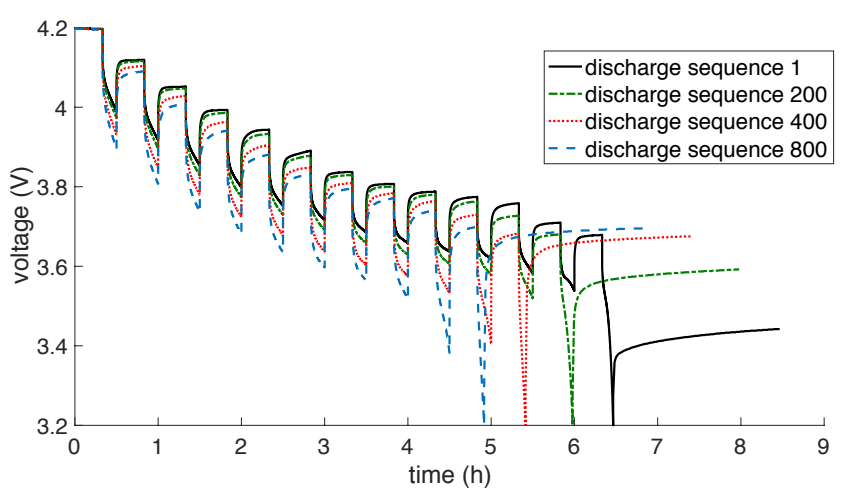

Figure 1: Battery discharge voltage Dynamics for different discharge sequences. In every sequence, a discharge current of $1 \mathrm{~A}$ is applied for 10 minutes after every 20 minutes of rest until the battery is completely discharged.

The diverse operating conditions of these devices make it difficult to model how and when the batteries are degrading, and when they need replacement. Indeed, most deployments operate devices continuously until they fail, and replace batteries or the entire device post-hoc. Having an effective mechanism for acquiring reliable information about the battery health state, i.e., battery capacity, would significantly facilitate the life-cycle management of these devices and ensure their operations with minimal disruptions.

Battery capacity is the most critical factor for long-term life-cycle management. Indeed, reduction in battery capacity causes problems such as, faster discharging of the batteries, frequent battery level corrections [8], and sudden shutdown of the devices [21]. Currently, reliably estimating battery capacity on IoT devices is unfortunately difficult as most IoT devices have limited means of acquiring such information. For example, higher-end smart devices can integrate a Coulomb counter-based fuel gauge [16] that can be used to estimate battery health upon a complete charge or discharge of the battery. Another option is to rely on a voltage-based fuel gauge, which can estimate capacity by modeling battery discharge curves [10]. However, these methods are unsuitable for most IoT devices due to their complexity and high cost.

The most common approach for IoT devices is to use power models that estimate both power drain and remaining capacity. For example, a simple linear model can count the energy spent per hour by emulating device characteristics with a simple battery 
model [3]. Another example is to use a model that accounts for energy consumption for the various operating steps of a device [15] Such models do not consider changes in the internal resistance characteristics of the battery over time, which affect the discharge rate of different operating loads. In fact, even two completely new batteries of the same model may have different internal resistance. Therefore, these models are either too simple, having poor accuracy $(60 \%)$, or require re-calibration with instrumentation of the sensing device [5]. Neither property is suitable for managing life-cycle of IoT devices deployed in the wild.

In this paper, we contribute by developing a novel method for estimating and predicting battery health through so-called V-edge dynamics. Our approach exploits the discharge load of IoT devices and identifies characteristics of discharge patterns to predict battery health. V-edges form in the battery's discharge curve as a result of a discharge load; see Figure 1 for an illustration. These edges result from the internal resistance characteristics of the battery, which increases through active usage as the battery ages. Hence, the characteristics of V-edges also change for a particular load.

We demonstrate the feasibility of our approach through a publicly available Lithium battery dataset [1]. Our evaluation results show that it is possible to estimate active battery usage time with the instantaneous voltage drop due to a discharge load with reasonable accuracy (over $80 \%$ ). This finding is very promising for the IoT devices having constant discharge load and short discharge period while operating in different weather conditions. We demonstrate that V-edge captures the internal resistance dynamics of a battery due to age, discharge load, and working conditions. Unlike other battery health estimation models for IoT devices [3, 4], our approach has better accuracy, and the sensor device only reads the battery voltage during a discharge load and computes V-edge once in a day, for example. With sufficient V-edge samples, the device can predict battery health. Alternatively, a sensor network management entity collects those and estimates the battery health of the IoT devices.

\section{BATTERY DATASET AND DEFINITIONS}

\subsection{Battery Dataset}

We consider a publicly available lithium battery dataset from NASA. The dataset primarily assisted in adapting the parameters of complex electrochemical battery models [1]. We use a subset of the measurements, which contains the cycling information of 8 lithiumion 18650 batteries with $2.2 \mathrm{Ah}$ initial capacity. The batteries were operated repeatedly, charging them with constant current and constant voltage $(\mathrm{CC} / \mathrm{CV})$ charging to $4.2 \mathrm{~V}$. The constant charging current was $2 \mathrm{~A}$. The batteries were then discharged to $3.2 \mathrm{~V}$ using a randomized sequence of discharging currents between $0.5-5 \mathrm{~A}$.

A complete discharge of a battery, i.e., 4.2-to-3.2V, is a sequence of random discharge current loads and resting events, $\mathrm{D}=\left\{d_{1}, r_{1}, d_{2}, r_{2}\right.$, $\left.d_{3}, r_{3}, \ldots, d_{n}, r_{n}\right\}$, where $d_{i} \& r_{i}$ represent a discharge and a rest event, respectively. The duration of each discharge event is one minute, and the average rest period is less than a second. Battery voltage, temperature, and discharge current are sampled after 1 second in an event along with a timestamp.

The discharge loads in a sequence follow the distributions presented in Table 1. Four batteries were discharged according to low-skew discharge distributions (LS), and the other four were

\begin{tabular}{|c|c|c|c|}
\hline \multicolumn{2}{|c|}{ Low Skew (LS) } & \multicolumn{2}{c|}{ High Skew (HS) } \\
\hline $0.5 \mathrm{~A}$ & $7.2 \%$ & $0.5 \mathrm{~A}$ & $2.0 \%$ \\
\hline $1.0 \mathrm{~A}$ & $14.8 \%$ & $1.0 \mathrm{~A}$ & $2.4 \%$ \\
\hline $1.5 \mathrm{~A}$ & $19.3 \%$ & $1.5 \mathrm{~A}$ & $3.6 \%$ \\
\hline $2.0 \mathrm{~A}$ & $21.6 \%$ & $2.0 \mathrm{~A}$ & $6.0 \%$ \\
\hline $2.5 \mathrm{~A}$ & $14.6 \%$ & $2.5 \mathrm{~A}$ & $9.2 \%$ \\
\hline $3.0 \mathrm{~A}$ & $10.0 \%$ & $3.0 \mathrm{~A}$ & $11.8 \%$ \\
\hline $3.5 \mathrm{~A}$ & $6.5 \%$ & $3.5 \mathrm{~A}$ & $17.2 \%$ \\
\hline $4.0 \mathrm{~A}$ & $4.0 \%$ & $4.0 \mathrm{~A}$ & $23.4 \%$ \\
\hline $4.5 \mathrm{~A}$ & $1.5 \%$ & $4.5 \mathrm{~A}$ & $19.4 \%$ \\
\hline $5.0 \mathrm{~A}$ & $0.5 \%$ & $5.0 \mathrm{~A}$ & $5.0 \%$ \\
\hline
\end{tabular}

Table 1: Distribution of discharge events with different discharge load currents on the batteries.

\begin{tabular}{|l|l|l|}
\hline Discharge Profile & Room Temperature & $40^{\circ} \mathrm{C}$ \\
\hline $\begin{array}{l}\text { Low Skew } \\
\text { (battery-id) }\end{array}$ & LS-RT & LS-40 ${ }^{\circ} \mathbf{C}$ \\
(bat-14,15) & $($ bat-23,24) \\
(discharge sequences) & $(1119,1124)$ & $(511,531)$ \\
(discharge events) & $(21510,22426)$ & $(20170,19468)$ \\
\hline High Skew & HS-RT & HS-40 ${ }^{\circ} \mathbf{C}$ \\
(battery-id) & (bat-19,20) & (bat-27,28) \\
(discharge sequences) & $(1307,1384)$ & $(605,611)$ \\
(discharge events) & $(15245,12739)$ & $(11022,9755)$ \\
\hline
\end{tabular}

Table 2: Four discharge profiles, corresponding batteries, and their cycling information, i.e., total discharge sequences and discharge events.

discharged according to the high-skew distribution (HS). The experiments were conducted in two temperatures: room temperature and $40^{\circ} \mathrm{C}$. Subsequently, there are four usage profiles, and we denote them, as shown in Table 2. Each profile can be mapped to the load characteristics of two kinds of IoT devices operating at two different environmental conditions, i.e., temperatures.

\subsection{Discharge events and V-edge}

Figure 2 characterizes the battery voltage drops across the internal resistance for a so-called pulsed discharge load of 1A for an equivalent battery model (Thevenin). Pulsed discharge loads correspond to profiles resulting from battery consuming operations that are performed repeatedly for a short duration of time with some time in between the repetitions, making them well suited for characterizing discharge profiles of typical IoT devices. For a particular discharge current, we notice that there is a sharp drop in the battery voltage as soon as the load begins. After that, the battery voltage reduces slowly during the load period. This is because the internal resistance $R$ is sensitive to this current. This sharp change in voltage is called the V-edge value, $V_{\text {edge }}$, which is $V_{\text {edge }}=R \times \Delta I=R \times I-R \times I_{0}$, where $I_{0}$ is the baseline current load, for example, a device is in the sleeping mode and draws current from the battery.

\section{V-EDGE AND BATTERY HEALTH}

The idea in our approach for battery health estimation is to construct models that relate $\mathrm{V}$-edge values and their characteristics with battery health through battery usage profiles. In this section, we first model V-edge values as a linear function of battery usage time according to discharge profiles. These models have median 


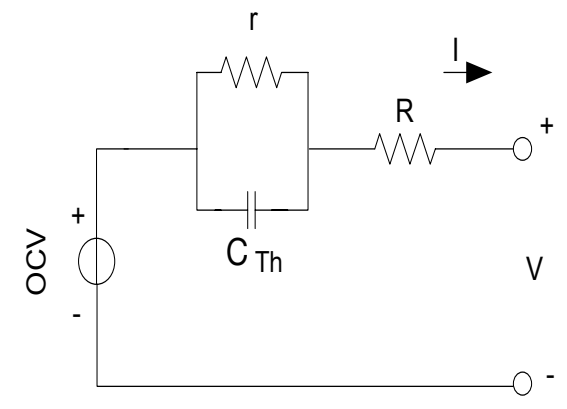

Battery Model (Thevenin Model)

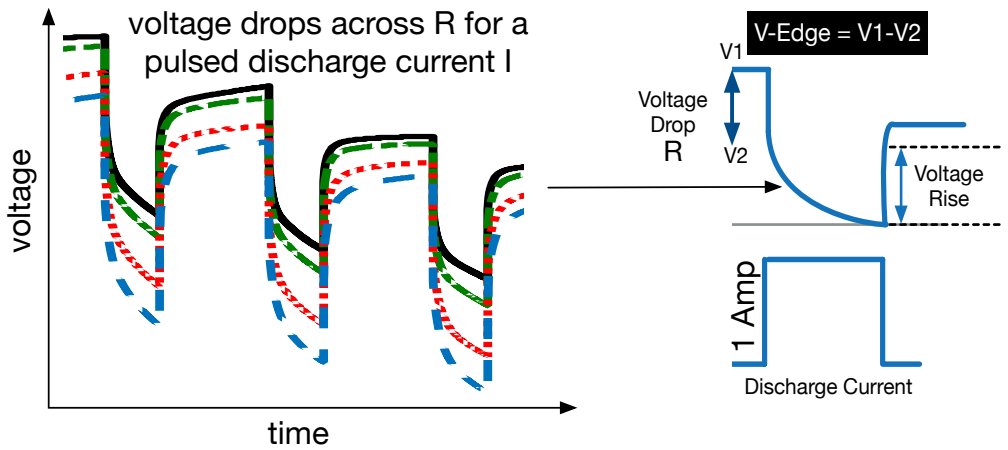

Figure 2: A Lithium battery model, voltage drops for a discharge load, and voltage drop across the internal resistance $\mathrm{R}$ (V-edge).

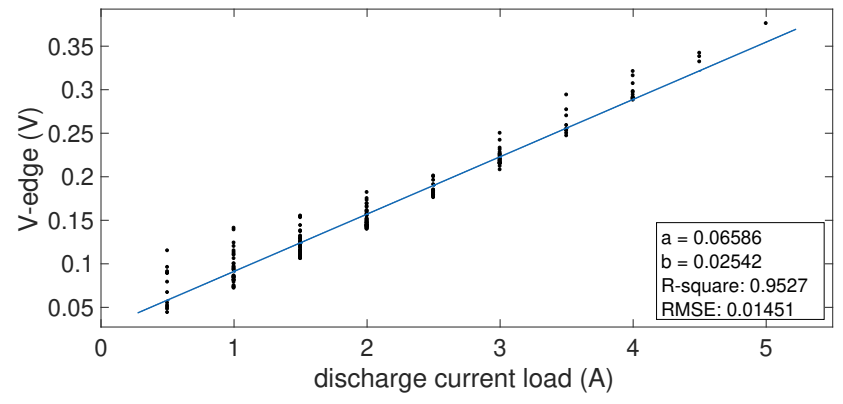

Figure 3: The Linear Regression fit of the V-edge values from Battery-15 according to various discharge currents.

errors of less than $20 \%$. We next associate the predicted battery usage time with battery capacity (Ah).

\subsection{Extracting V-edges}

The discharge voltage sampling frequency is $1 \mathrm{~Hz}$, and thus, the voltage sequence during a discharge event is $V d_{i}:\left\{V_{1}, V_{2}, \ldots, V_{60}\right\}$ ordered in increasing time. Similarly, a rest event is set of two voltages $V r_{i}:\left\{V_{1}, V_{2}\right\}$. We compute a V-edge by subtracting the first discharge voltage from the last voltage of previous resting event in a discharge sequence, i.e, $V i_{\text {edge }}=V r_{i-1}(2)-V d_{i}(1)$. The time delta between these two voltage updates is less than a second. This ensures that we compute the instantaneous voltage drop. In this dataset, there were no baseline loads during the discharge experiments, and thus, $V_{\text {edge }}=R \times \Delta I=R \times I$.

In the dataset, the discharge load period is one minute. In practice, an embedded or IoT device may have shorter discharge periods. The advantage of using V-edge is that it can be characterized within a short period of time. Xu et al. used lithium-ion batteries of smartphones and showed that the relation between the discharge load current and V-edge is linear [23]. The authors applied such a relation to model the runtime power consumption of mobile devices. On the contrary, we explore the relation between V-edge and battery health for specific discharge current loads. As we are investigating cylindrical lithium-ion batteries, we first verify the behavior of V-edges. Figure 3 shows that such linear relation also holds for the 18650 lithium batteries considered in our experiments.
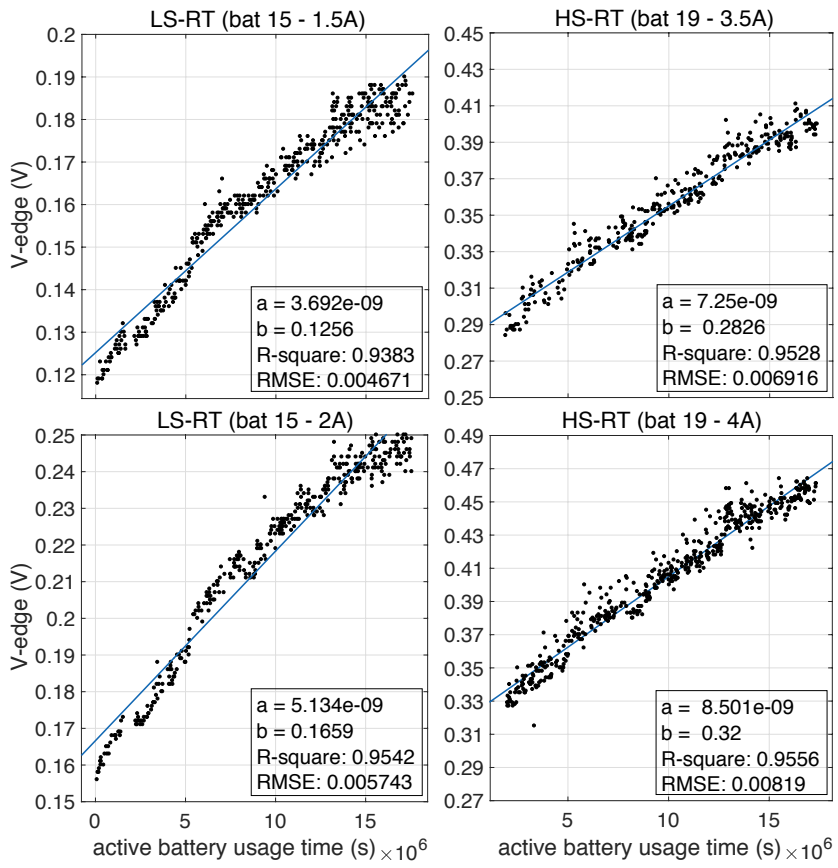

Figure 4: The Linear Regression fits of the V-edge values for different discharge profiles at room temperature.

\subsection{Predicting Active Battery Usage Time}

We next demonstrate how the discharge profiles in Table 2 affect $\mathrm{V}$-edge over time. We take the first two V-edge values from two consecutive discharge events from each sequence and classify those according to the discharging loads for all discharge sequences. We then construct linear regression models for the discharge loads presented in Table 1.

Figures 4 and 5 show the fitted linear regression models of Vedge values against the age of the battery for both user profiles at room temperature and at $40^{\circ} \mathrm{C}$ temperature, respectively. The coefficient, $a$, of the regression model, is the inclination of the regression line, and $b$ is the intercept. The presented coefficients are with $95 \%$ confidence bounds.

We observe that the coefficients vary according to the discharge load among similar profiles, and higher discharge currents exhibit 

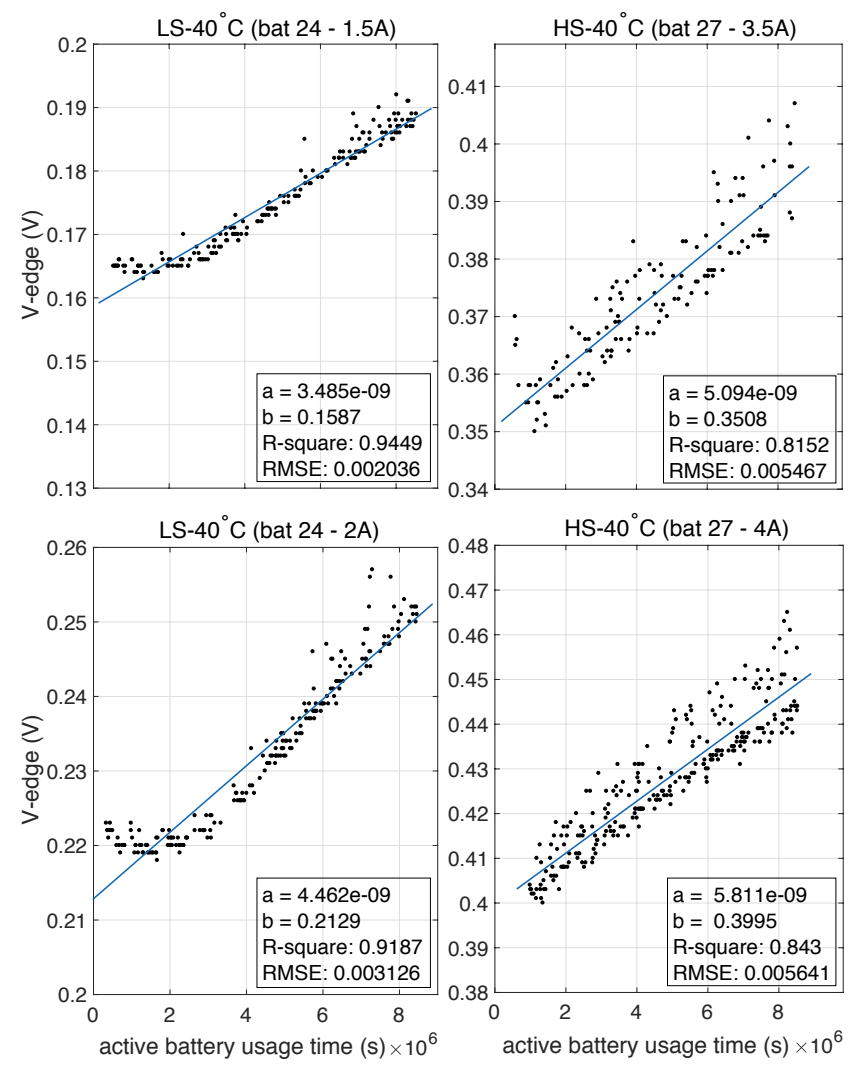

Figure 5: The Linear Regression fits of the V-edge values for different discharge profiles at $40^{\circ} \mathrm{C}$.

steeper slopes and higher intercepts. Comparing the models in Figure 4 and Figure 5 on the basis of ambient temperature, we see that the baseline $\mathrm{V}$-edge value (intercept) is higher at $40^{\circ} \mathrm{C}$ than at room temperature for similar discharge loads. In all profiles, the slope also increases as the discharge load increases.

In the plots, we determine goodness of linear fit using $R^{2}$ values. For the LS profiles in Figures 4 and 5, the $R^{2}$ values are above 0.9 , indicating that variance in V-edge values is almost around its mean irrespective of the ambient temperature. The $R^{2}$ values for the HS profiles at $40^{\circ} \mathrm{C}$ are slightly lower at 0.85 . The results thus imply that the performance of the model is somewhat sensitive to temperature, with higher operating temperatures decreasing model fit. Nevertheless, the results are encouraging as the model fit is excellent in all cases, ranging between $85-96 \%$.

\subsection{Performance Evaluation}

The previous section considered V-edge values from the first two consecutive discharge events for constructing models. We next find the V-edge values from longer-term behavior by assessing the next three successive discharge events for the same battery. We randomly select 100 from all such V-edges, predict battery age, and evaluate the performance of the models of four profiles. Figure 6 demonstrates that the models can predict the active usage time of the batteries with more than $80 \%$ accuracy. The batteries of HS profiles have median errors of less than $20 \%$.
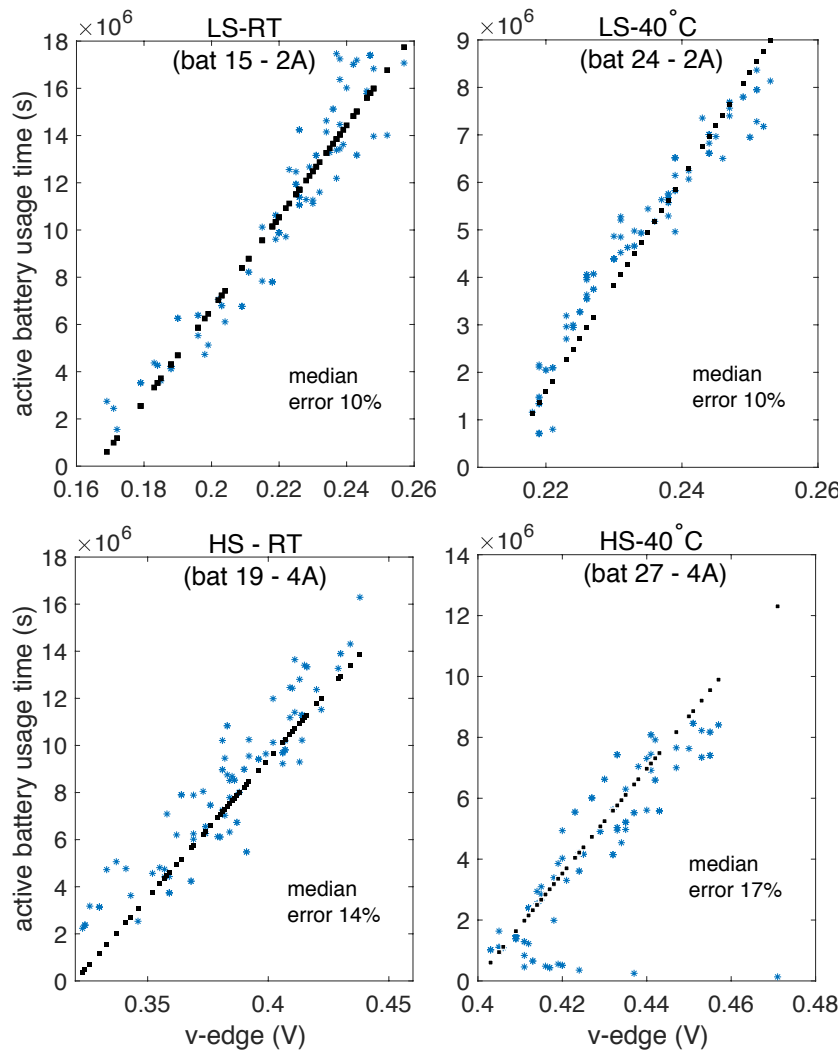

Figure 6: Performance of profile specific regression models.

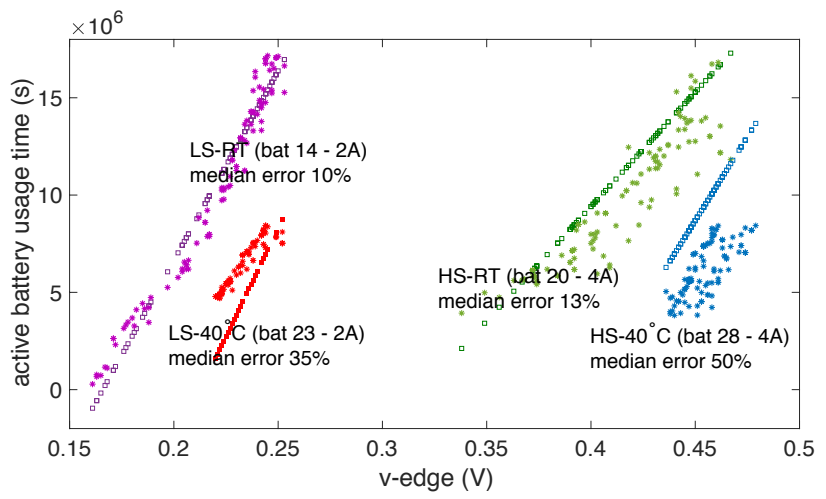

Figure 7: Performance of the regression models in Figure 4 and Figure 5 for the remaining four batteries.

We next analyze the performance of the same profile-specific models on the other four batteries, i.e., bat $14,20,23$, and 28 . Note that the V-edge values of these batteries were not used in constructing the earlier models. In other words, we want to predict the active battery usage time of another set of IoT devices having similar operating conditions before deployment. We randomly take 100 V-edge values and use the corresponding models to predict active usage time. Figure 7 demonstrates the actual values and prediction. It further shows that such models perform better with the batteries operating at room temperature. The errors increase for LS $-40^{\circ} \mathrm{C}$ 
and HS- $40^{\circ} \mathrm{C}$ batteries compared to the batteries of the same profile. Such performance is expected, as the internal resistance across the battery samples of having the same capacity can vary. We additionally constructed similar models for the lower discharge loads, e.g., 0.5 and 1.0A. These models have had less than $20 \%$ errors.

To summarize, our models are capable of accurately explaining different types of discharge loads, with the best results coming under normal operating temperatures. As the temperature or battery discharge profile changes, the performance degrades slightly, but the accuracy of our models remain consistently high, reaching over $80 \%$. Overall, this is a very encouraging result, suggesting that V-edges are accurate at estimating parameters, which in turn can be used to estimate battery capacity.

\subsection{Discharge Profiles and Battery Health}

Battery capacity decreases with usage, and the pace of changes depends on the operating conditions. After every fifty charging and discharging, a reference discharging was performed with $1 \mathrm{~A}$ load on the batteries. A reference discharge is a series of pulsed discharge events at $1 \mathrm{~A}$ for 10 minutes, followed by 20 minutes of resting period, as shown in Figure 1. We compute the health of the batteries by integrating the currents over time for a reference discharge sequence, which gives the battery capacity in Ah. Since there are only a few data points for such estimates, we construct the regression models for four batteries from four profiles and present the fits in Figure 8.

The models have $R^{2}$ values higher than $95 \%$, and we can utilize the predicted active battery usage time values in Figure 6 as inputs to these models to get the health estimates in Ah. The remaining four batteries have had very good linear fits.

\section{DISCUSSIONS}

Our results indicate that the dynamics of V-edge correlate well with the usage profiles of the batteries. Consequently, the prediction models also generalize. It is even possible to use the profile-specific models to predict the active usage time of another new battery, having a similar capacity and discharge load, with reasonable accuracy at room temperature. In this section, we highlight future research directions using V-edge dynamics.

Dynamic Discharge Profiles. In practice, the internal resistance of a battery also should vary with the state of charge. Therefore, complex models are required to capture the combined effect of usage and the state of charge on V-Edge. Furthermore, the present models apply to the devices with fixed discharge loads. Smartphones, smartwatches, and other similar devices may have dynamic discharge loads, and their usage may correspond to the whole distribution of the discharge loads presented in Table 1. Subsequently, the discharge load distributions as a whole and predicting the battery health of smart devices is our future research direction.

Predicting Device Performance. The complex usage profiles come with additional challenges related to automated battery management functionality. For example, smart devices have been reported to shut down even when they claim to have around $15-20 \%$ of battery remaining. The culprit for this behavior relates to sudden voltage drops, which take the battery voltage below a cut-off threshold that is used to determine when the shut down the device [21].

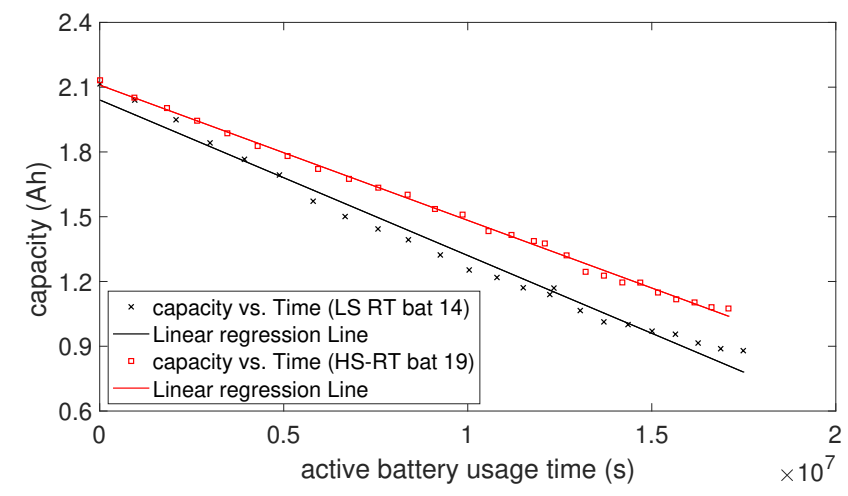

(a) Room Temperature

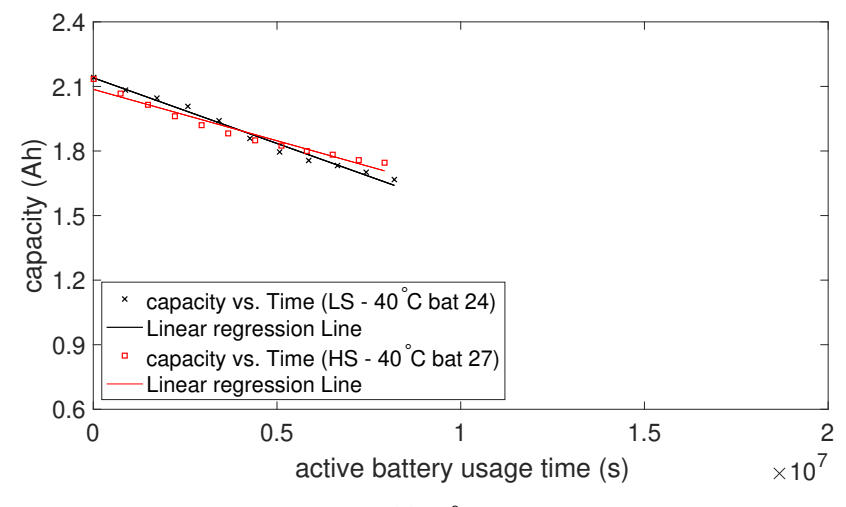

(b) $40^{\circ} \mathrm{C}$

Figure 8: Linear regression models for battery active usage time and capacity degradation in $\mathrm{mAh}$.

V-edges and other related voltage information make it possible to predict such events and develop intelligent power management schemes that can avoid this type of sudden shutdowns.

Calibrating Power Models. In Figure 3, we notice that the relation between V-edge and discharge current is linear. This relation has been previously used to develop fast power consumption models for smartphones [23]. Nevertheless, in previous research, it was assumed that such a relation is static. Our work demonstrates that V-edge increases as the battery health degrades, which implies that there is a concept drift. Thus power models need continuous calibration. V-edges provide a possible way to detect and assess the drift, and can potentially be used to re-calibrate the power models.

\section{RELATED WORK}

Model-based health estimation is the traditional approach for battery capacity modeling. These can be summarized as electrochemical models [18], equivalent circuit-based models [6], and analytical models [20] depending on the nature of measurements. These models tend to be highly sensitive to the modeling environment, measurement technology, and battery design and materials. Hence these approaches are unsuited for most IoT devices.

Charging voltage curves can be used to assess battery health [22, 24]. For example, Lu et al. proposed an approach that models battery degradation as a function of four geometrical features of the charging voltage curves [14]. The performance of these approaches 
heavily relies on the charging algorithm, which varies from one device to another [9]. Hoque et al. used the duration of charging to reach the maximum voltage to estimate the battery health [7].

Data-driven methods estimate battery life using machine learning, e.g., support vector machines [17], Gaussian process regression [13], or multiple linear regression [19]. These models tend to suffer from limited generality and require several input parameters that are difficult to capture on heterogeneous IoT deployments.

\section{CONCLUSIONS}

We demonstrated that the instance voltage drops due to discharge load can be utilized to construct battery life prediction models for various IoT devices. These models are simple and can be used for devices with limited battery instrumentation to predict their usage time. Our approach works best when the models are constructed under similar discharge loads and operating temperatures, but even when different profiles and temperatures are used for modeling, we reach around $80 \%$ accuracy. We further discussed how to improve the power consumption models and predict the device performance with old batteries. For the practical deployment, our method only requires knowing the capacity of a fresh battery and the discharge current of the device, which can be acquired from datasheets or measured during the design phase of the IoT networks. When highly accurate measurements are required, our approach benefits from temperature measurements. These can be the battery temperature measurements which are available from most fuel gauges or use an environmental temperature sensor as a proxy. At present, we are integrating our models for the battery health management of battery-powered air quality monitoring sensors.

\section{ACKNOWLEDGEMENTS}

We thank our shepherd Dr. Josiah Hester and anonymous reviewers for their valuable comments. We thank the NASA team for producing such an excellent dataset. This study would have been difficult without this dataset. This research is partly supported by the National Research Foundation of Korea (NRF), ICT (2017R1A2B3010504, 2017M3C4A7065963, 2017M3C4A7066473) and by the Academy of Finland project CBAI (Crowdsourced Battery Optimization AI for a Connected World) grant no 1319017.

\section{REFERENCES}

[1] B. Bole, C. Kulkarni, and M. Daigle. Adaptation of an electrochemistry-based li-ion battery model to account for deterioration observed under randomized use. page 9, 092014.

[2] F. Dressler, M. Mutschlechner, B. Li, R. Kapitza, S. Ripperger, C. Eibel, B. Herzog, T. Hönig, and W. Schröder-Preikschat. Monitoring bats in the wild: On using erasure codes for energy-efficient wireless sensor networks. ACM Trans. Sen. Netw., 12(1):7:1-7:29, Feb. 2016

[3] W. Dron, S. Duquennoy, T. Voigt, K. Hachicha, and P. Garda. An emulation-based method for lifetime estimation of wireless sensor networks. In Proceedings of the 2014 IEEE International Conference on Distributed Computing in Sensor Systems, DCOSS '14, pages 241-248, Washington, DC, USA, 2014. IEEE Computer Society.

[4] A. Dunkels, F. Osterlind, N. Tsiftes, and Z. He. Software-based on-line energy estimation for sensor nodes. In Proceedings of the 4th Workshop on Embedded Networked Sensors, EmNets '07, pages 28-32, New York, NY, USA, 2007. ACM.

[5] X. Fafoutis, A. Elsts, A. Vafeas, G. Oikonomou, and R. Piechocki. On predicting the battery lifetime of iot devices: Experiences from the sphere deployments. In Proceedings of the 7th International Workshop on Real-World Embedded Wireless Systems and Networks, RealWSN'18, pages 7-12, New York, NY, USA, 2018. ACM.

[6] H. He, X. Zhang, R. Xiong, Y. Xu, and H. Guo. Online model-based estimation of state-of-charge and open-circuit voltage of lithium-ion batteries in electric vehicles. Energy, 39(1):310-318, 2012.
[7] M. A. Hoque, M. Siekkinen, J. Koo, and S. Tarkoma. Full charge capacity and charging diagnosis of smartphone batteries. IEEE Transactions on Mobile Computing, 16(11):3042-3055, Nov 2017.

[8] M. A. Hoque and S. Tarkoma. Sudden drop in the battery level?: Understanding smartphone state of charge anomaly. In Proceedings of the Workshop on PowerAware Computing and Systems, HotPower '15, pages 26-30, New York, NY, USA, 2015. ACM.

[9] M. A. Hoque and S. Tarkoma. Characterizing smartphone power management in the wild. In Proceedings of the 2016 ACM International foint Conference on Pervasive and Ubiquitous Computing: Adjunct, UbiComp '16, pages 1279-1286, New York, NY, USA, 2016. ACM.

[10] T. Instruments. bq27621-g1 system-side fuel gauge with dynamic voltage correlation. Technical report.

[11] P. Kamalinejad, C. Mahapatra, Z. Sheng, S. Mirabbasi, V. C. Leung, and Y. L. Guan. Wireless energy harvesting for the internet of things. IEEE Communications Magazine, 53(6):102-108, 2015.

[12] E. Lagerspetz, S. Varjonen, F. Concas, J. Mineraud, and S. Tarkoma. Megasense: Megacity-scale accurate air quality sensing with the edge. In Proceedings of the 24th Annual International Conference on Mobile Computing and Networking, MobiCom '18, pages 843-845, New York, NY, USA, 2018. ACM.

[13] D. Liu, J. Pang, J. Zhou, and Y. Peng. Data-driven prognostics for lithium-ion battery based on gaussian process regression. In Proceedings of the IEEE 2012 Prognostics and System Health Management Conference (PHM-2012 Beijing), pages 1-5. IEEE, 2012.

[14] C. Lu, L. Tao, and H. Fan. Li-ion battery capacity estimation: A geometrical approach. Journal of power sources, 261:141-147, 2014.

[15] B. Martinez, M. Montón, I. Vilajosana, and J. D. Prades. The power of models: Modeling power consumption for iot devices. IEEE Sensors fournal, 15(10):57775789 , Oct 2015.

[16] Maxim. MAX17047/MAX17050, ModelGauge m3 Fuel Gauge. Technical report, 2014.

[17] M. A. Patil, P. Tagade, K. S. Hariharan, S. M. Kolake, T. Song, T. Yeo, and S. Doo. A novel multistage support vector machine based approach for li ion battery remaining useful life estimation. Applied Energy, 159:285-297, 2015.

[18] S. Sankarasubramanian and B. Krishnamurthy. A capacity fade model for lithiumion batteries including diffusion and kinetics. Electrochimica Acta, 70:248-254, 2012.

[19] K. Severson, P. Attia, N. Jin, N. Perkins, B. Jiang, Z. Yang, M. Chen, M. Aykol, P. Herring, D. Fraggedakis, M. Bazant, S. Harris, W. Chueh, and R. Braatz. Datadriven prediction of battery cycle life before capacity degradation. Nature Energy, 4:1-9, 052019.

[20] F. Sun, X. Hu, Y. Zou, and S. Li. Adaptive unscented kalman filtering for state of charge estimation of a lithium-ion battery for electric vehicles. Energy, 36(5):35313540, 2011.

[21] Y. Sun, L. Kong, H. Abbas Khan, and M. G. Pecht. Li-ion battery reliability - a case study of the apple iphone ${ }^{\circledR}$. IEEE Access, 7:71131-71141, 2019.

[22] Z. Wang, S. Zeng, J. Guo, and T. Qin. Remaining capacity estimation of lithium-ion batteries based on the constant voltage charging profile. PloS one, 13(7):e200169, 2018.

[23] F. Xu, Y. Liu, Q. Li, and Y. Zhang. V-edge: Fast self-constructive power modeling of smartphones based on battery voltage dynamics. In Proceedings of the 10th USENIX Conference on Networked Systems Design and Implementation, nsdi'13, pages 43-56, Berkeley, CA, USA, 2013. USENIX Association.

[24] Y. Zheng, C. Qin, X. Lai, X. Han, and Y. Xie. A novel capacity estimation method for lithium-ion batteries using fusion estimation of charging curve sections and discrete arrhenius aging model. Applied Energy, 251:113327, 2019. 